\title{
The heparan sulfate and its diverse biological activities
}

\author{
IWONA KAZNOWSKA-BYSTRYK
}

Department of Laboratory Diagnostics, Medical University of Lublin, Chodzki 1, 20-093 Lublin, Poland

\begin{tabular}{l}
\hline ARTICLE INFO \\
\hline Received 01 Dec 2014 \\
Accepted 09 Dec 2014 \\
\hline
\end{tabular}

Keywords:

heparan sulfate, glycosaminoglycans, proteoglycans, atherosclerosis, lipoproteins.

\begin{abstract}
Heparan sulfate (HS) is one of the most common glycosaminoglycan (GAG) in mammals. It is composed of relatively simple disaccharide subunits, which, by further modification, such as sulfation and epimerization, potentially offer huge diversity in biological function. GAG chains of different length, different patterns of sulfation, and other modifications, depending on location, generate unique forms. Due to polyanion charges, these compounds can interact with other molecules, such as proteins, cytokines, chemokines and growth factors, both on the cell surface and inside the extracellular matrix. These interactions serve protective and storage functions for the compounds, safeguarding them from proteolysis. In this way, HS is involved in numerous signaling pathways, and in growth and differentiation processes. Disrupted interactions between the HS and growth factors, cytokines or other proteins have been observed in various disorders, among these Alzheimer's disease, epilepsy, atherosclerosis, diabetes, and cancer processes. Detailed knowledge of these relationships at the molecular level will allow researchers to understand the mechanisms underlying these disorders and enable the development of effective therapeutic strategies.
\end{abstract}

\section{INTRODUCTION}

Heparan sulfate (HS) is a glycosaminoglycan (GAG) consisting of sulfated repeating disaccharide units composed of $\mathrm{N}$-acetylated or $\mathrm{N}$-sulphated glucosamine units and glucuronic or iduronic acid. HS, as well as heparin, are anticoagulant glycosaminoglycans, and are widely distributed in human tissues. The HS chains undergo diverse modifications, including N-deacetylation, $\mathrm{N}$ - or O-sulfation and epimerization in the Golgi apparatus by the various enzymes in a tissue-specific manner $[1,7,11]$. Modifications in chains number and saccharide size provide important variability with regard to biological functions. Sulfate groups present in these GAGs give them a polyanionic character, defining not only the structure and hydrodynamic volume, but also the diverse interactions with the various signaling factors. Usually HS, like other GAGs, is covalently attached to the core protein, generating a special class of biomolecules heparan sulfate proteoglycans (HSPG) $[1,11]$.

The HSPGs are located in the extracellular matrices and on cell surfaces of various kinds of human tissues. Based upon their location, HS can be grouped three ways: HS found on the cell surface, in the extracellular matrices (ECM) and in the intracellular granules $[1,7]$.

Corresponding author

e-mail: iwona.kaznowska-bystryk@umlub.pl
- Membrane HSPG, such as syndecan, glypican and betaglycan, cooperate with integins and receptors to facilitate cell-matrix components attachment, cell-cell interactions and cell motility. Some of these can act as co-receptors for the various tyrosine- kinase type growth factor receptors, and some act as endocytic receptors - which are relevant in lipoprotein metabolism in the liver.

- Serglycin is found within the secretory vesicles, and plays a role in the packaging of the granular contents and in regulating coagulation, wound repair and host defence.

- Extracellular matrix HSPG (perlecan, agrin and collagen XVIII) collaborate with other ECM components to modulate basement membrane structure and cell migration $[1,7,10,15]$.

The HS chains contain highly sulfated domains, partially sulfated and non-sulfated domains, which are transitional. The domains with highly sulfated residues are formed by consecutive clusters of sulfated disaccharides and are degraded by extracellular sulfatases $[1,15]$.

HS is one of the most highly negatively charged extracellular molecules, and the interactions of many positivelycharged proteins with HS have little specificity beyond charge interactions [7]. HS can interact with different molecules of the ECM, transmitting signals promoting cell migration, inducing adhesion, and bringing about the 
rearrangement of cell cytoskeleton. HSPG can bind growth factors, matrix proteins, cytokines and chemokines, protecting them against proteolysis $[7,11,15,21]$. In this way, it enables the retention of these factors which can be subsequently liberated by selective degradation of the HS chains. Moreover, it facilitates the formation of the chemokine or morphogen gradients essential for leukocyte recruitment and homing, and cell development. Most crucial determinants of interaction between HS and protein on HS chain are the density and position of the sulfate groups, epimerization, the length of HS chain, and its 3D conformation [21].

HS have essential roles in urinary, circulatory, nervous, skeletal and immune systems, as well as embryological development and wound repair $[1,15,16]$. The products of HS degradation by the action of heparanase are directly engaged in tumor advancement and metastasis [11].

\section{Heparan sulfate in central nervous system}

$\mathrm{HS}$, as well as CS, are the most common GAGs present in the central nervous system (CNS). They regulate diverse functions in the CNS by interacting with a number of matrix proteins, growth factors and cell surface molecules $[15,17]$. The functionality of HS is, to a large degree, dictated by the sulfation patterns present on their chains as found within progenitor cells, including the neuroepithelial layer of the CNS. This GAG accumulates and supplies growth factors to the respective stem cell surface receptors [17].

The role of HSPGs is dependent on the developmental stage of the CNS. Syndecans and glypicans are the most common HSPGs in the CNS. During CNS development, Glipican-1 is prominently expressed in the actively mitotic areas of neurogenesis. However, Syndecan-3 is mostly expressed during the glial cell differentiation, myelination, and formation of neuronal connections. Its expression decreases in adult neurons, and it is mainly found in the axons. Both glypicans and syndecans are closely associated with neurite outgrowth, which was confirmed in research that employed HS-degrading enzymes which resulted in a disturbance of the axonal path finding [17].

The sulfotransferases, which act by sulfating residues at specific positions, regulate the temporal variations in the HS chains. The affinity of HS-ligand complexes is significantly dependent on subtle structural changes and a diversity in the influence of these interactions on the neurons. Although some evidence alludes to HSPG having some importance in plasticity, it remains unclear which sulfation patterns are required for influencing this. As has been shown in the animal model, the lack of endosulfatases induces deficiencies in spatial learning and deficits in synaptic plasticity within the hippocampus [17].

HS is widely studied for the role it plays in neurological disorders such as Alzheimer's disease (AD). AD comes about by way of an accumulation of plaques - aggregated amyloid- $\beta(A \beta)$ peptides, and neurofibrillary tangles in the brain cortex $[5,17]$. Accumulation and aggregation of the toxic forms of $A \beta$ in the extracellular space has a crucial effect in the pathogenesis of $\mathrm{AD}$, and is responsible for cognition and memory dysfunctions [5]. Research has found highly sulfated HS chains to be co-localized with amyloid- $\beta$ plaques. Hence, it has been suggested that the highly sulfated domains could be involved in the sequestration of vascular endothelial growth factor (VEGF) within amyloid plaques, and in the induction of vascular damage in AD through disturbance in the supply of pro-angiogenic growth factors. Of note, abnormal angiogenesis could induce neurovascular uncoupling and synaptic dysfunction [5]. It has also been shown that HS is important for the regulation of Alzheimer's $\beta$-secretase (BACE1) by binding to BACE-1 and inhibiting the cleavage of the amyloid precursor protein, and, thus, altering the protection of the fibril against microglial phagocytosis $[5,17]$.

HSPGs such as neurocan, versican, and tenascin, also play a significant role in some gliomas, as these interact with the factors that promote glioma growth and invasion. Research shows that sulfation of GAGs can alter the behavior of glioma cells; for example, reduction of sulfation results in lower levels of cell proliferation [17].

\section{Heparan sulfate in circulatory system}

HS takes part in the formation of tunica intima and tunica media neointima, and can be found in the glycocalyx layer [19]. The glycocalyx is a layer lining the vascular endothelium, acting as a barrier between it and the blood stream, and exerts various roles in vessel wall processes and blood homeostasis $[4,14,20]$. In vasculature, HSPG represents approximately $50-90 \%$ of the total amount of PG present in glycocalyx, but this amount is variable. HS and CS occur together with a typical ratio of 4:1 in the vascular endothelium glycocalyx [14]. As the HS chains contain numerous specific binding sites for plasma-derived molecules, small chain modifications, which may vary in time and under different stimuli, can have great functional consequences. The diversity of HS sulfation patterns and its effect on the specific binding of the molecules and modulation of protein function suggest that conditions that diminish glycocalyx thickness, or modulate HS sulfation patterns and charge, are likely to modulate vascular permeability and modify specific protein binding. Due to the presence of numerous highly sulfated HS groups, the glycocalyx is characterized by a net negatively charged surface to the blood and the neutralization of this layer induces a growth in the albumin uptake by endothelial cells, and, hence, a rise in the permeability [14].

The glycocalyx role in the interaction between leucocytes and vessel walls seems to be dual: firstly, it harbors the adhesion molecules: ICAM, VCAM, P-selectin; and secondly, it suppresses the adhesion of leucocytes to these particles. Application of heparitinase upon the HS chains in glycocalyx leads to an increase in leukocyte adhesion to this layer in a dose-dependent manner. Damage to the glycocalyx layer plays a key role in several vascular disorders, e.g.: during ischemia, atherosclerosis and diabetes [14]. It has been shown that a local reduction in the glycocalyx HS content is associated with increased sub-endothelial accumulation of LDL at the atherogenic region [20].

Among the over 20 various PGs identified in the vascular wall, perlecan is one of the major extracellular HSPGs that is located in both the intima and media of blood vessels [16]. Perlecan's core protein has three HS side chain binding sites in domain I, and one added site in domain V [7]. In the different animal species, its significance in atheroscerosis is not 
consistent in the experimental models, because, in monkeys, it is only visible in the advanced phase, while, in mice, it is evident in both the early and the advanced phases [13].

HS has been found to be intimately involved in regulating the release of nitric oxide (NO) as a mechano-shear sensor for NO release in vascular homeostasis [20]. It is suggested that significant HSPGs associated with the membranes, such as glypican, mediate the immediate NO response to shear stress, while syndecan takes part in the remodeling of the response for a longer period [19].

According to the "response to retention" hypothesis, PGs are one of the major ECM molecules which interact with apoB-containing lipoproteins (LP) in the subendothelial matrix, doing so by direct interaction, or via bridging molecules, such as secretory phospholipase A2 (sPLA2), lipoprotein lipase (LPL) or apoE $[4,9,18]$. The direct reaction occurs mainly through electrostatic binding between the negatively charged carboxyl and sulfate groups on the HS chains of PG, and the positively charged lysine and arginine residues of apo B100 [9, 13]. Histological studies of human vessels reveal firm co-localization between biglycan and apo B100 LP. Of note, the LP retention process displays self-accelerating features. Moreover, what is more important for the atherosclerosis development is the bulk load of LP deposition in the wall, rather than the specific type of LP retained. Additionally, cytokines take part in this initial stage of atherogenic cascade, and enhance the local production of PG with the high binding affinity for LP; it is the degree of sulfation, rather than the position of the sulfate groups on GAG, which determines LDL binding [13]. The main determinants of retained LPs within the vessel wall seem to be the LP characteristics and the capacity of the vessel wall to bind LP [4]. Many factors of the cellular environment influence the sulphation and elongation of the GAG chains, including ECM components, growth factors, fatty acids and oxidized LDL. Therefore, they affect the atherogenicity of the HSPG $[4,8]$.

HS has also been involved in the stabilization and presentation of hepatic lipase on cell surfaces and in the transport of LPL to the luminal surface of the endothelium [8]. Thus, LPL has binding sites for both LP and HSPG. As a consequence, macrophage-derived LPL raise the vessel wall affinity for LP, creating a solid bridge between PG and LP [4]. Furthermore, HSPG found in the lipid rafts acts as the LPL docking station: it serves as a platform for interaction with TG-rich lipoproteins (TRL) at the endothelial cell surface. In hepatocytes, which internalize the remnant particles, the most abundant HSPG is Syndecan 1, a crucial molecule for the binding and clearance of TG-rich remnant lipoproteins. The key factor in hepatic remnant clearance is the specific sulfation forms, not the total negative charge [2].

\section{Heparan sulfate in renal function}

The main kidney glycosaminoglycan is HS, and it has a double function in renal physiology: to sustain a fixed negative charge at the filtration barrier and to take part in the glycocalix layer which covers the outer surface of the podocytes [3].
The glomerular basement membrane (GBM) is the layer between two cells: podocytes and fenestrated endothelial cells. It has an average thickness of 300-400 nm, and the gel-like structure is comprised approximately $90 \%$ of water [6]. The GBM is composed predominantly of ECM molecules: laminin, type IV collagen, nidogen and HSPG. Of the last, agrin is the major HSPG. They together create a meshwork, giving both the GBM's charge-selective and size-selective properties. As with all basement membranes, the GBM especially exhibits high net negative charge, and HSPGs (such as agrin and perlecan) significantly contribute towards this negative charge [12].

The notion of the negative charge of the GBM being essential in engendering a filtration barrier (for example, to albumin), has been in acceptance for many years. Nevertheless, the once fully accepted assumption of charge selectivity is now controverted on the grounds of diverse recent research. For example, the in vivo decrease in the anionic charge of GBM by the heparanase infusion (which results in the removal of HS side chains from the proteoglycans) does not cause proteinuria. What is more, the removal of agrin from the GBM by podocyte selective knock-out of agrin results in a significant reduction of the negative charges in the GBM without the effect of albuminuria. Thus, the role of GBM is now deemed to be minor, whereas the importance of the endothelial glycocalyx has been emphasized $[6,12]$.

The glycocalyx covering glomerular endothelium consists of a network of proteoglycans with GAG side chains carrying a negative charge. These make up an extracellular matrix. Only recently has its prominent confining function been acknowledged. Moreover, it is now known that the systemic dysfunction of the endothelium manifests itself by impaired NO production, raised endothelial permeability and a prothrombotic tendency [12].

Recent data indicates that the overlying glycocalyx has a more important role in the development of proteinuria. An enzymatic degradation by heparinase reduces the thickness of the endothelial cell glycocalyx, modifying its negative charge, and the effect is related to albuminuria. According to the Steno hypothesis, albuminuria in type I and II diabetes may reflect the kidney manifestations of a systemic dysfunction of the endothelium. Indeed, it has been established that hyperglycemia may increase the permeability of the glycocalyx independently of its volume changes $[6,12,14]$.

Since HSPG can modulate cell activities by their interaction with various matrix molecules, e.g. proteins, cytokines, adhesion molecules, and growth factors, they can also interact with the complement factors. The concept of using GAG as complement inhibitors in different immunemediated renal diseases was postulated for many years. This indicates the possible role of PG as a docking platform for complement activation. What is important, the fine structure of GAG determines whether the complement might be activated by preferential binding of complement factors, or be muted by binding of regulatory proteins. Targeted interaction with GAG might thus cause the complement activation at the kidney level [21]. 


\section{SUMMARY}

Heparan sulfate (HS) is one of the most widespread GAG in mammals. It is composed of the relatively simple disaccharide subunits which can achieve a great level of diversification due to modifications, such as sulfation or epimerization. Different chain lengths, varied sulfation patterns and other modifications impart to the eventual molecules, a unique form, dependent on their location. Due to polyanion charges, these compounds can interact with other molecules, such as proteins, cytokines, chemokines and growth factors, both on the cell surface and inside the extracellular matrix. These interactions serve protective and storage functions for the compounds, safeguarding them from proteolysis. This way, HS participates in the numerous signalling pathways of the development and differentiation processes. The impaired interactions between HS and growth factors, cytokines or other proteins can be observed in different disorders, such as Alzheimer's disease, epilepsy, atherosclerosis, diabetes and carcinogenic processes. A precise recognition of the discussed interactions on a molecular level will facilitate understanding of the mechanisms responsible for these disorders, and allow the design of therapeutic strategies.

\section{REFERENCES}

1. Bishop J. R., Schuksz M., Esko J.D.: Heparan sulphate proteoglycans fine-tune mammalian in physiology. Nature, 446, 1030, 2007.

2. Dallinga G.M. et al.: The metabolism of triglyceride-rich lipoproteins revisited: New players, new insight. Atherosclerosis, 211, 1-8, 2010.

3. De Muro P. et al.: Urinary glycosaminoglycans composition in chronic glomerulonephritis. J Nephrol., 18, 154-160, 2005.

4. Fogelstrand P., Boren J.: Retention of atherogenic lipoproteins in the artery wall and its role in atherogenesis. Nutrition, metabolism \& Cardiovascular Diseases, 22, 1-7, 2012.

5. Hosono-Fukao T. et al.: Heparan Sulfate subdomains that are degraded by sulf accumulate in cerebral amyloid $\beta$ plaques of Alzheimer's disease. Am J Pathol., 180, 2056-2067, 2012.
6. Jefferson A., Shankland S.J., Pichler R.H.: Proteinuria in diabetic kidney disease: A mechanistic viewpoint. Kidney International, 74, 22-36, 2008.

7. Kirn-Safran C., Farach-Carson M.C., Carson D. D.: Multifunctionality of extracellular and cell surface heparan sulfate proteoglycans. Cell. Mol. Life Sci., 66, 3421-3434, 2009.

8. Kolset S.O., Salmivirta M.: Cell surface heparan sulfate proteoglycans and lipoprotein metabolism. Cellular and molecular life sciences, 56, 857, 1999.

9. Lähdesmäki K. et al.: Acidity and lipolysis by group V secreted phospholipase A(2) strongly increase the binding of apoB-100containing lipoproteins to human aortic proteoglycans. Biochim Biophys Acta, 1821, 257, 2012.

10. Madonna R., De Caterina R.: Potential roles of vessel wall heparan sulfate proteoglycans in atherosclerosis. Vascular Pharmacology, 62, 3, 120-122, 2014.

11. Malavaki Ch. J. et al.: Heparan sulfate: biological significance, tools for biochemical analysis and structural characterization. Biomed. Chromatogr., 25, 11, 2011.

12. Miner J. H.: The Glomerular Basement Membrane. Exp Cell Res., 318(9): 973-978, 2012.

13. Nakashima Y., Wight T.N., Sueishi K.: Early atheroslcerosis in humas: role of diffuse intimal thickening and extracellular matrix proteoglycans. Cardiovascular research, 79, 14, 2008.

14. Reitsma S. et al.: The endothelial glycocalyx: composition, functions, and visualization. Pflugers Arch-Eur J Phisiol, 454, 345-359, 2007.

15. Sarrazin S., Lamanna W. C., Esko J.D.: Heparan Sulfate Proteoglycans. Cold Spring Harb Perspect Biol., 3;a004952, 2011.

16. Segev A., Nili N., Strauss B.H.: The role of perlecan in arterial injury and angiogenesis. Cardiovascular Research, 63, 603, 2004.

17. Swarup V. P. et al.: Sugar glues for broken neurons. BiolMolecular Concepts, 4, 3, 233-257, 2013.

18. Tannock L. R., King V. L.: Proteoglycan mediated lipoprotein retention: A mechanism of diabetic atherosclerosis. Reviews in Endocrine \& Metabolic Disorders, 9, 289, 2008.

19. Tarbell J. M. Tarbell: Shear stress and the endothelial transport barrier, Cardiovasc Res., 87, 320, 2010.

20. van den Berg B. M., Spaan J. A., Vink H.: Impaired glycocalyx barrier properties contribute to enhanced intimal low-density lipoprotein accumulation at the caroid artery bifurcation in mice. Pflugers Arch - Eur J Physil., 457: 1199-1206, 2009.

21. Zaferani A. et al.: Heparin/heparan sulphate interactions with complement - a possible target for reduction of renal function loss? Nephrol Dial Transplant, 0: 1-9, 2013. 